\title{
Programme de soutien «Recherche sur les services de santé»
}

\author{
Michael Röthlisberger ${ }^{a}$, Hermann Amstad ${ }^{b}$ \\ ${ }^{a}$ Dr sc. nat., ancien responsable du ressort Sciences, ASSM, Berne; ${ }^{b}$ Dr méd., Secrétaire général, ASSM, Berne
}

Les références se trouvent sous www.bullmed.ch $\rightarrow$ Numéro actuel ou $\rightarrow$ Archives $\rightarrow 2017 \rightarrow 3$

\section{Symposium} 2017

Le $1^{\text {er }}$ mars 2017 I'ASSM organise le $5^{\mathrm{e}}$ symposium sur la recherche sur les services de santé à Berne. Programme et inscription: assm.ch/fr/agenda
Grâce au lancement du Programme National de Recherche PNR 74 «Système de santé» par le Fonds national suisse (FNS) en automne 2015 [1] ${ }^{*}$, et à un nouveau programme de soutien de la fondation Recherche suisse contre le cancer [2] élaboré dans le cadre de la Stratégie nationale contre le cancer, la recherche sur les services de santé dispose enfin de fonds substantiels. Le programme de soutien «Recherche sur les services de santé» de l'Académie Suisse des Sciences Médicales (ASSM) avait posé les premières bases dans ce domaine.

La recherche sur les services de santé examine, entre autres, les effets des interventions médicales dans les conditions du quotidien, c'est-à-dire le transfert des résultats de la recherche clinique dans la réalité des cabinets médicaux, des hôpitaux et des institutions de soins. Dès 1985, l'ASSM avait posé les premiers jalons du réseau de la recherche sur les services de santé en Suisse: avec son programme «Recherches et réalisations en médecine appliquée» (RRMA), elle soutenait des projets de recherche du domaine de la médecine de premier recours [3] avec 200000 CHF par an. Grâce à la Fondation Gottfried et Julia Bangerter-Rhyner, l'ASSM a pu lancer en 2011 le programme de soutien «Recherche sur les services de santé». Après la première phase de sélection, l'Office fédéral de la santé publique (OFSP) a chargé l'ASSM d'élaborer un concept pour le «renforcement de la recherche sur les services de santé en Suisse» [4]. Presque simultanément, le Conseil fédéral s'est fixé comme priorité explicite de son agenda de politique de la santé «Santé 2020" des mesures visant à assurer et à améliorer la qualité des soins. Le programme de soutien a donc été lancé au moment opportun.

\section{Objectifs, moyens et instruments d'encouragement}

La Fondation Bangerter a mis un million de francs suisses par an à la disposition du programme de soutien de 2012 à 2016. Conformément à la convention, le programme doit permettre:

- à moyen ou à long terme de développer durablement les compétences et les infrastructures dans le domaine de la recherche sur les services de santé en Suisse;

- de réaliser des projets de recherche de haut niveau dans le domaine de la recherche sur les services de santé;

- de promouvoir la relève scientifique dans ces domaines;

- de donner à ce domaine une impulsion décisive lui permettant, à l'avenir, d'acquérir des fonds en concurrence avec d'autres domaines de recherche.

La notion de "recherche sur les services de santé» recouvre un large éventail de questions de recherche. Le programme de soutien a défini la recherche sur les services de santé selon le modèle de Pfaff [5] comme «un domaine de recherche interdisciplinaire qui décrit et explique de façon causale les soins de santé et la prise en charge des malades et leurs conditions cadres dans le but de développer des concepts de soins scientifiquement fondés, qui soutient l'implémentation de nouveaux concepts de soins et qui évalue l'efficacité des structures et des procédures des services de santé dans la pratique quotidienne».

Le programme de soutien proposait trois différents instruments d'encouragement aux requérants: le soutien de projets (montant maximal $200000 \mathrm{CHF} / \mathrm{an}$ pendant trois ans au maximum), des bourses individuelles pour des séjours à l'étranger (40000 CHF) et des financements de départ ("Seed Money») pour des projets pilotes (25000 CHF).

\section{Critères d'évaluation et procédures}

Une commission interdisciplinaire constituée de 15 experts était responsable de l'évaluation des requêtes. Les requêtes ont d'abord été examinées par le secrétariat général de l'ASSM et le président de la commission. Les projets conformes aux objectifs ont ensuite été soumis à deux experts et évalués selon les critères suivants:

- Evaluation de la personne (max. 8 points): formation, parcours, environnement, performances scientifiques, compétences spécifiques en relation avec le projet; 
- Evaluation du projet (max. 16 points): valeur scientifique, actualité du projet, originalité du sujet, adéquation de la méthodologie, faisabilité du projet, connexion à un réseau, durabilité, return on investment.

Les points donnés par les deux experts ont été additionnés et les projets classés selon le nombre de points. Les projets ayant atteint un total de 38 points ou plus (48 points au max.) ont été discutés en détail lors de la séance d'évaluation. Par ailleurs, les experts ont également examiné les projets qui n'ont pas atteint un total $>38$ points, mais dont les évaluations individuelles divergeaient fortement.

Dans les projets jugés dignes de soutien, des adaptations budgétaires ont pu être réalisées ou des conditions formulées. Sur la base de ces sélections, une proposition a été soumise à la Fondation Bangerter. Le Conseil de fondation s'est réservé le droit d'accepter ou de refuser ces propositions; celles-ci n'ont toutefois jamais été refusées.

\section{Récapitulatif des requêtes déposées}

$\mathrm{Au}$ total, 344 requêtes ont été déposées dans les cinq phases de sélection; 259 d'entre elles ont été jugées conformes à l'objectif et évaluées. La part de requêtes non conformes à l'objectif a nettement diminué entre la première et la dernière sélection: $32 \%$ vs $14 \%$.

Parmi les requêtes conformes à l'objectif figuraient 200 soutiens de projets, 49 financements de départ et

Le programme de soutien «Recherche sur les services de santé» en un coup d'œil.

\begin{tabular}{|c|c|}
\hline Durée: & 2012-2016 \\
\hline $\begin{array}{l}\text { Nombre de requêtes } \\
\text { soumises: }\end{array}$ & 344 \\
\hline $\begin{array}{l}\text { Nombre de requêtes } \\
\text { conformes à l'objectif: }\end{array}$ & 259 \\
\hline $\begin{array}{l}\text { Nombre de requêtes } \\
\text { soutenues: }\end{array}$ & $\begin{array}{l}46 \text { ( } 35 \text { promotions de projets, } 8 \text { financements de départ, } \\
2 \text { bourses, } 1 \text { soutien d'une manifestation) }\end{array}$ \\
\hline Montant sollicité: & 33 millions $\mathrm{CHF}$ \\
\hline Montant octroyé: & 4,4 millions $\mathrm{CHF}$ \\
\hline $\begin{array}{l}\text { Provenance des } \\
\text { requêtes soumises: }\end{array}$ & $\begin{array}{l}\text { Hôpitaux universitaires et cantonaux } 42 \% \\
\text { Universités } 30 \% \\
\text { Hautes écoles spécialisées } 19 \% \\
\text { Autres } 9 \%\end{array}$ \\
\hline $\begin{array}{l}\text { Provenance des } \\
\text { requêtes soutenues: }\end{array}$ & $\begin{array}{l}\text { Hôpitaux universitaires et cantonaux } 17 \% \\
\text { Universités } 72 \% \\
\text { Hautes écoles spécialisées } 7 \% \\
\text { Autres } 4 \%\end{array}$ \\
\hline $\begin{array}{l}\text { Thèmes et partenaires } \\
\text { des symposiums: }\end{array}$ & $\begin{array}{l}\text { 2012: médecine de premier recours; Collège de médecine } \\
\text { de premier recours } \\
\text { 2013: oncologie; SAKK } \\
\text { 2014: interprofessionnalité; Hautes écoles spécialisées } \\
\text { de la santé } \\
\text { 2015: "Less is more»; SSPH+ } \\
\text { 2017: I'avenir de la recherche sur les services de santé; } \\
\text { FNS, SSPH+ }\end{array}$ \\
\hline
\end{tabular}

9 bourses (l'une des requêtes concernait le financement des coûts d'organisation d'un événement; cette possibilité n'était envisagée que lors de la première phase de sélection).

$42 \%$ des requérants principaux venaient d'hôpitaux universitaires ou cantonaux, 30\% d'entre eux d'universités et $19 \%$ de hautes écoles spécialisées (10\% d'autres horizons). 89 des 213 requérants principaux avaient un titre académique de professeur ou de PD, 164 d'entre eux avaient au moins un titre de Docteur, et 9 d'entre eux avaient suivi une formation postgraduée de Master of Public Health (MPH). Comme prévu, la majorité des requérants principaux travaillaient dans les cinq grandes villes universitaires (Zurich: 61; Berne: 44; Bâle: 37; Lausanne: 33; Genève: 24). 12 requêtes provenaient de Winterthour et 11 de Saint-Gall. 5 requêtes, dans lesquelles des institutions suisses ont joué un rôle décisif, ont été soumises depuis l'étranger.

Dans le classement par disciplines, la médecine venait en première position (133 requêtes), suivie des sciences infirmières (34), de la santé publique et de la médecine préventive (33), et de l'économie (de la santé) (19). 110 projets ont pu être clairement attribués au secteur ambulatoire, tandis que 71 projets du secteur stationnaire ont été examinés. Dans 52 autres projets, les soins ont été considérés à un niveau systémique.

La somme globale demandée s'élevait à 33 millions de francs suisses.

\section{Caractéristiques des projets soutenus}

La commission d'experts avait proposé de soutenir 46 des 259 requêtes soumises (18\%): il s'agissait dans 35 cas de soutiens de projets, dans 8 cas de financements de départ, dans 2 cas de bourses et dans 1 cas de frais d'organisation d'une manifestation. Les 46 projets soutenus ont été soumis par 39 requérants différents. Parmi les 46 requêtes soutenues, 33 provenaient d'universités (72\%), 8 d'hôpitaux (17\%), 3 de hautes écoles spécialisées (7\%) et 2 d'autres institutions (4\%). Presque tous les requérants principaux avaient au moins un titre de Docteur (36 respectivement 92\%), 21 un titre de Prof./PD (54\%). La plupart des projets soutenus venaient de Zurich (16), de Bâle (11), de Berne (8), de Lausanne (5) et de Genève (3); autres: 3 . La majorité des projets encouragés traitaient de problèmes rencontrés dans le secteur ambulatoire (25). Les projets des domaines stationnaires (5) et les projets relatifs au système (7) étaient moins nombreux. Le montant des soutiens s'élevait à 4422717 CHF au total. En moyenne, 150000 CHF ont été attribués aux projets qui ont bénéficié d'un soutien; un montant fixe de 25000 CHF était prévu pour les financements de départ respectivement 
de 40000 CHF pour les bourses. A ce jour, 70\% des projets soutenus lors des deux premières sélections ont donné lieu à des publications dans des Peer-reviewed Journals; les chiffres concernant les sélections ultérieures ne sont pas encore disponibles.

Le taux de $18 \%$ de projets encouragés dans le cadre d'un programme de soutien scientifique correspond aux attentes. De même, le fait que la plupart des projets soutenus émanent du contexte universitaire n'a rien de surprenant. En revanche, on ne s'attendait pas à des taux de réussite aussi divergents entre les projets des instituts universitaires et ceux des hôpitaux ou des hautes écoles spécialisées: alors que 30\% des projets émanant d'universités ont été soutenus, seuls $12 \%$ des projets venant d'hôpitaux et $6 \%$ de ceux venant de hautes écoles spécialisées ont bénéficié d'un soutien. Les experts ont évoqué à plusieurs reprises l'insuffisance de la qualité scientifique des projets des hautes écoles spécialisées comme motif de refus (en dépit du grand intérêt du sujet du projet). Le taux de réussite très bas des projets émanant d'hôpitaux peut être attribué, par exemple, à leur focus sur des problèmes très spécifiques de la prise en charge (avec un nombre restreint de cas) ou au potentiel limité de généralisation des résultats attendus.

Le fait que 43 des 46 projets soutenus aient été soumis par un investigateur venant de l'une des cinq grandes villes universitaires suisses reflète l'importance du lien universitaire d'un projet quant à ses chances de succès. Le chiffre élevé des projets du secteur ambulatoire qui ont été soumis (110 ou $42 \%$ ) et soutenus ( 25 ou $54 \%$ ) reflète la tendance générale à une "ambulatorisation" de la médecine. Dans ce sens, la recherche sur les services de santé peut offrir un soutien au développement de nouveaux modèles.

\section{Symposium de la recherche sur les services de santé en Suisse}

Dr Katrin Crameri, ancienne responsable du ressor «Sciences» de l'ASSM et actuellement à l'OFSP, a largement contribué à la rédaction de cet article. Dr Michael Röthlisberger travaille depuis septembre 2016 à la Ligue suisse contre e cancer.

Correspondance: Dr méd. Hermann Amstad Secrétaire général ASSM Maison des Académies

Laupenstrasse 7

CH-3001 Berne

h.amstad[at]samw.ch
«Less is More» avec la Swiss School of Public Health Les présentations scientifiques ont été complétées par des sessions de posters. Les posters - soumis sur la base d'une procédure d'appel d'offres et sélectionnés par le comité scientifique - ont reflété l'ensemble des aspects de la recherche sur les services de santé en Suisse. Environ 600 personnes ont participé aux quatre symposiums et plus de 200 posters y ont été présentés. L'annonce pour le prochain symposium est parue récemment, thème: PNR 74 «Système de santé», avec le FNS (cf. www.samw.ch/fr/agenda).

\section{Les objectifs du programme de soutien ont-ils été atteints?}

Au fil des années, le programme de soutien «Recherche sur les services de santé» a été reconnu par une communauté scientifique de plus en plus importante. De même, la popularité du symposium correspondant n'a cessé d'augmenter. Le nombre relativement élevé de requêtes (environ 50 requêtes conformes aux objectifs par an) dépassait nettement les chiffres actuels des requêtes pour d'autres instruments d'encouragement de l'ASSM, tels que le programme de soutien «Recherche en soins palliatifs» ou le Fonds Käthe Zingg-Schwichtenberg pour des projets dans le domaine de l'éthique médicale. L'activité dans le domaine de la recherche sur les services de santé en Suisse se reflète également dans le nombre élevé de projets soumis dans le cadre du PNR 74 «Système de santé» et du programme de soutien de la Recherche suisse contre le cancer. Ces constatations permettent d'affirmer que les objectifs du programme de soutien ont été largement atteints $[6,7]$.

\section{Perspectives}

L'ASSM s'engage pour un encouragement ciblé de la recherche dans des domaines encore peu ou pas établis. Compte tenu de l'évolution des dernières années et notamment de l'instauration du PNR 74, le développement de la recherche sur les services de santé n'est plus du ressort de l'ASSM.

Le PNR sélectionne actuellement les projets de la première mise au concours. D'autres mises au concours ne sont pas prévues pour le moment. La fondation Recherche suisse contre le cancer attribue chaque année, de 2016 à 2020, un million de francs dans le cadre du «Programme de renforcement de la recherche sur les services de santé en oncologie». Le cinquième symposium de la recherche sur les services de santé en Suisse, qui se déroulera le $1^{\text {er }}$ mars 2017, sera consacré à la question de savoir comment le soutien de la recherche sur les services de santé peut être garanti à l'avenir. 